\title{
RECURSOS INTANGÍVEIS E A RELAÇÃO ENTRE DESEMPENHO SOCIAL E DESEMPENHO FINANCEIRO EM EMPRESAS BRASILEIRAS
}

\author{
INTANGIBLE RESOURCES AND THE RELATIONSHIP \\ BETWEEN SOCIAL PERFORMANCE AND FINANCIAL \\ PERFORMANCE IN BRAZILIAN COMPANIES
}

\section{RECURSOS INTANGIBLES Y LA RELACIÓN ENTRE EL DESEMPEÑO SOCIAL Y EL DESEMPEÑO FINANCIERO EN EMPRESAS BRASILEÑAS}

Simone Barakat

Docente da Universidade Anhembi Morumbi, Brasil

Doutora em Administração pela Universidade de São Paulo, Brasil

simonebarakat@usp.br

Keysa Manuela Cunha de Mascena

Docente da Universidade de Fortaleza, Brasil

Doutora em Administração pela Universidade de

São Paulo, Brasil

keysamascena@usp.br

\section{Greici Sarturi}

Docente da Universidade Federal de Santa Maria, Brasil

Doutora em Administração pela Universidade de São Paulo, Brasil

greicisarturi@hotmail.com

Pedro Takenouchi

Mestre em Administração pela Universidade de São Paulo, Brasil

pedro_issao@yahoo.com.br
Contextus

ISSNe 2178-9258

Organização: Comitê Científico Interinstitucional Editor-Chefe: Diego de Queiroz Machado

Avaliação: double blind review pelo SEER/OJS

Recebido em 01/08/2019

Aceito em 11/09/2019

Versão final aceita em 25/09/2019

http://dx.doi.org/10.19094/contextus.v17i3.41743

\section{RESUMO}

A relação entre o desempenho social corporativo e o desempenho financeiro corporativo (CSP-CFP) é estudada como uma relação direta, e pouco se conhece sobre as variáveis que podem ser mediadoras desta relação. Diante desse contexto, o objetivo desta pesquisa é testar se os recursos intangíveis são mediadores da relação CSP-CFP em empresas no Brasil. Analisou-se uma amostra de 230 empresas listadas em 2013 na Bolsa, Brasil, Balcão (B3). As hipóteses foram testadas por meio de regressões múltiplas pelo Método dos Quadrados Mínimos (MQO). Os resultados confirmam a hipótese de que há uma relação positiva entre CSP e CFP e que os recursos intangíveis são mediadores dessa relação. O estudo contribui para constatar, na amostra analisada no contexto brasileiro, que as empresas com desempenho social superior, que levou ao desenvolvimento de recursos intangíveis, obtiveram desempenho financeiro superior.

Palavras-chave: desempenho social corporativo; desempenho financeiro corporativo; recursos intangíveis; stakeholders; índice de sustentabilidade empresarial. 


\begin{abstract}
The relationship between corporate social performance and corporate financial performance (CSP-CFP) is studied as a direct relationship, and little is known about the variables that may mediate this relationship. Given this context, the objective of this research is to test whether intangible resources mediate the CSP-CFP relationship in companies in Brazil. A sample of 230 companies listed in 2013 on the Bolsa, Brasil, Balcão (B3) was analyzed. The hypotheses were tested by multiple regressions using the Least Squares Method (OLS). The results confirm the hypothesis that there is a positive relationship between CSP and CFP and that intangible resources mediate this relationship. The study contributes the finding, in the sample analyzed in the Brazilian context, that companies that presented superior social performance, which led to the development of intangible resources, obtained superior financial performance.

Keywords: corporate social performance; corporate financial performance; intangible resources; stakeholders; corporate sustainability index.
\end{abstract}

\title{
RESUMEN
}

La relación entre el desempeño social corporativo y el desempeño financiero corporativo (CSP-CFP) se estudia como una relación directa, y se sabe poco acerca de las variables que pueden mediar en esta relación. Dado este contexto, el objetivo de esta investigación es probar si los recursos intangibles median la relación CSP-CFP en las empresas de Brasil. Se analizó una muestra de 230 empresas que cotizaban en 2013 en la Bolsa, Brasil, Balcão (B3). Las hipótesis se probaron utilizando regresiones múltiples y el Método de Mínimos Cuadrados (OLS). Los resultados confirman la hipótesis de que existe una relación positiva entre CSP y CFP y que los recursos intangibles median esta relación. El estudio contribuye al encontrar, en la muestra analizada en el contexto brasileño, que las empresas que presentaron un desempeño social superior, lo que llevó al desarrollo de recursos intangibles, obtuvieron un desempeño financiero superior.

Palabras clave: desempeño social corporativo; desempeño financiero corporativo; recursos intangibles; stakeholders; índice de sostenibilidad corporativa.

\section{INTRODUÇÃO}

As questões sociais e ambientais tornaram-se assuntos presentes nas agendas dos gestores e nas discussões teóricas no campo da administração (AGUINIS; GLAVAS, 2012; CHENG; IOANNOU; SERAFEIM, 2014; DE BENEDICTO et al., 2013; MELO et al. 2018). Sua importância está tanto na valorização da empresa frente ao mercado e seus diversos stakeholders, quanto na possível relação entre essas questões e o desempenho financeiro (HILLMAN; KEIM, 2001; MAZZIONI et al., 2013; HUSTED; ALLEN; KOCK, 2015; RIBEIRO, 2016).

Nas três últimas décadas, ampliou-se o volume de pesquisas que buscam analisar a relação entre o desempenho social corporativo (corporate social performance - CSP) e o desempenho financeiro corporativo (corporate financial performance - CFP). A CSP pode ser interpretada como o amplo conjunto de estratégias e práticas que a empresa desenvolve para lidar e criar relacionamentos com os seus inúmeros stakeholders e com o meio ambiente (WADDOCK, 2004).

A relação positiva entre CSP e CFP tem argumentos teóricos favoráveis e contrários. Alguns autores consideram que adotar práticas socialmente responsáveis melhora o 
desempenho financeiro da empresa (AGUINIS; GLAVAS, 2012; MCWILLIAMS; SIEGEL, 2011), uma vez que o bom relacionamento com os múltiplos stakeholders gera vantagens competitivas (RUSSO; FOUTS, 1997; RUF et al., 2001; HUSTED; ALLEN; KOCK, 2015). Outros autores defendem como negativa a relação entre CSP e CFP, pois consideram que ações de responsabilidade social sejam fontes de altos custos (FRIEDMAN, 1970; LAZONICK; O'SULLIVAN, 2000) e que os custos gerados por essas práticas nunca serão recuperados pela empresa (KARNANI, 2011). Na literatura, há também argumentos de que a relação não exista ou não possa ser detectada, devido ao grande número de variáveis intervenientes (MADORRAN; GARCIA, 2016; NELLING; WEBB, 2009; ULLMANN, 1985). Em meio a argumentos divergentes, pesquisas que realizaram meta-análises sobre os resultados de estudos empíricos mostraram predomínio de estudos que encontraram uma relação positiva entre CSP e CFP (MARGOLIS; WALSH, 2003; ORLITZKY; SCHMIDT; RYNES, 2003).

Algumas pesquisas buscaram compreender outros aspectos dessa relação, como a direção da causalidade (WADDOCK; GRAVES, 1997) ou a linearidade da relação (BARNETT e SALOMON, 2012). Porém, falham em não considerar variáveis que possam mediar a relação (MCWILLIAMS; SIEGEL, 2000; SURROCA; TRIBÓ; WADDOCK, 2010). Além disso, os trabalhos publicados são focados, quase exclusivamente, em empresas norte-americanas e europeias (SURROCA; TRIBÓ; WADDOCK, 2010).

Poucos estudos encontrados buscaram testar a relação CSP-CFP em empresas do Brasil. Registram-se os trabalhos de Abreu et al. (2009); Beato, Souza e Parisotto (2009); Machado, Machado e Corrar (2009); Crisóstomo, Freire e Vasconcellos (2011); Orellano e Quiota (2011), Souza-Cunha e Samanez (2013), Freguete, Nossa e Funchal (2015) e Grecco, Geron e Grecco (2017). Esses estudos têm encontrado resultados pouco conclusivos. Além de encontrar resultados divergentes, as pesquisas não consideraram o efeito de variáveis mediadoras.

A relação CSP-CFP não se configura como clara e direta (LUO et al., 2015; MADORRAN; GARCIA, 2016), conforme vem sendo tratada nos estudos em amostras brasileiras. A CSP poderá ter efeito positivo no desempenho financeiro da empresa na medida em que possibilite o desenvolvimento de recursos intangíveis, caso contrário poderá ter efeitos neutros e até mesmo negativos (HILLMAN; KEIM, 2001; SURROCA; TRIBÓ; WADDOCK, 2010; TURRA et al., 2015). Esses recursos intangíveis podem incluir inovação, capital humano, reputação e cultura organizacional, que para esses autores são variáveis mediadoras entre CSP e CFP. Ou seja, a CSP primeiramente tem um efeito positivo no desenvolvimento de 
recursos intangíveis, que por sua vez têm um desenvolvimento positivo no desempenho financeiro (SURROCA; TRIBÓ; WADDOCK, 2010).

Diante desse contexto, o objetivo desta pesquisa é testar se os recursos intangíveis são mediadores da relação CSP-CFP em empresas no Brasil. Para isso, utilizaram-se dados secundários de 230 empresas listadas em 2013 na bolsa de valores, mercadorias e futuros de São Paulo - Bolsa, Brasil, Balcão (B3). Para investigação da CSP, foram consideradas as empresas que participam do Índice de Sustentabilidade Empresarial (ISE).

O presente trabalho apresenta uma contribuição empírica ao investigar no contexto brasileiro um tema amplamente investigado em estudos que utilizam bases internacionais para verificar a CSP. A análise em diferentes contextos é importante uma vez que estudos anteriores encontraram efeitos significantes do contexto do país nos achados sobre CSP (ABREU; CUNHA; BARLOW, 2015; FRÍAS-ACEITUNO; RODRÍGUEZ-ARIZA; GARCÍASÁNCHEZ, 2013). Este estudo também reforça a literatura sobre a relação CSP-CFP ao considerar uma variável capaz de mediar a relação, pois críticas à literatura alegam que os modelos analisados têm variáveis omitidas (LUO et al., 2015; MCWILLIAMS; SIEGEL, 2011) e apresentam relações de causalidade que podem ser espúrias (RUF et al., 2001; SURROCA; TRIBÓ; WADDOCK, 2010).

\section{REFERENCIAL TEÓRICO}

\subsection{Desempenho Social Corporativo (CSP)}

A CSP é um construto que, segundo Carroll (1979), inclui as dimensões de responsabilidade social, as questões sociais e a responsividade social. A CSP envolve uma definição básica do conceito e dos tipos de responsabilidade social, um entendimento e enumeração das questões de responsabilidade social, e uma especificação da filosofia (ou estratégia) para responder a essas questões. Ressalta-se que, para Carroll (1979), a filantropia está incluída em uma das quatro perspectivas da responsabilidade social: econômica, legal, ética e filantrópica. Ou seja, interpreta-se que a filantropia não é o núcleo central da CSP. Ullmann (1985) define CSP como a resposta às demandas sociais, definição que se assemelha à responsividade social de Carroll (1979). 
Waddock e Graves (1997) consideram a CSP um construto multidimensional com comportamentos que variam por meio de entradas, processos e saídas. As entradas podem incluir investimentos em equipamentos de controle de poluição; como processos pode-se considerar tratamento de minorias, relacionamento com consumidores, produtos produzidos; e as saídas podem incluir o impacto na comunidade, entre outros.

Em obra posterior, Carroll (2000) reforça que CSP pode ser entendida como uma avaliação abrangente do desempenho social e não uma avaliação isolada do desempenho da empresa em relação uma questão social específica ou a um stakeholder. Para o autor, ao estudar a CSP deve-se considerar a relação da empresa com pelo menos quatro grupos de stakeholders principais: empregados, clientes, acionistas e comunidade.

A CSP é considerada por Griffin (2000) como uma interação contínua entre a organização e seus stakeholders, e vice-versa. Se esse conceito pudesse ser substituído por outro que o represente, seria definido pela expectativa da sociedade e pelo processo iterativo de respostas das empresas para essas expectativas. Por sua vez, Barnett (2007) descreve a CSP como um retrato do desempenho social geral de uma empresa em um determinado ponto no tempo; um resumo da postura social agregada da empresa.

Muitos outros autores tentaram definir e mensurar CSP, o que por vezes é objetivo de crítica. Para posicionar o presente estudo, considera-se o conceito de CSP de Waddock (2004), definido como o amplo conjunto de estratégias e práticas que a empresa desenvolve para lidar e criar relacionamentos com os seus inúmeros stakeholders e com o meio ambiente.

\subsection{Relação entre CSP e CFP}

A maioria dos estudos empíricos sobre CSP concentra-se na análise da relação entre CSP e CFP (AGUINIS; GLAVAS, 2012). Devido à grande quantidade de estudos que analisaram essa relação, alguns pesquisadores consolidaram os resultados dos estudos, como Orlitzky, Schmidt e Rynes (2003) que fizeram uma meta-análise de 52 estudos; Margolis e Walsh (2003), que revisaram 127 estudos entre 1972 e 2002; e Boaventura, Silva e Bandeira de Mello (2012), que revisaram 58 artigos no período de 1996 a 2010.

Orlitzky, Schmidt e Rynes (2003) chegaram à conclusão de que há uma relação positiva entre ambos os desempenhos. A meta-análise dos autores sugere que a CSP e CFP são mutuamente reforçadas, e que uma pode prever a outro e vice-versa. Os autores sugerem que 
as empresas podem desenvolver relações benéficas com os diversos grupos de stakeholders e serem financeiramente recompensadas.

Margolis e Walsh (2003) classificaram os estudos em quatro categorias: relação positiva, relação negativa, relação não significante ou relações mistas. Além disso, agruparam os estudos que consideraram a CSP como variável independente (explicativa) ou como dependente. Os autores encontraram que a maioria dos estudos utiliza a CSP como variável independente, e que a relação CSP-CFP é predominantemente positiva. Boaventura, Silva e Bandeira de Mello (2012) apresentaram em seu estudo que 39 artigos publicados usaram a CSP como variável independente, 14 artigos usaram como variável dependente e 5 analisaram das duas formas. Quanto à relação entre CSP e CFP, verificaram que 38 artigos encontraram uma relação positiva; 11 artigos uma relação negativa e 18 artigos uma relação neutra ou não relacionada.

Com relação aos fatores que levam ao uso da CSP ou CFP como variável dependente, Waddock e Graves (1997) apresentam argumentos teóricos que denominam como slack resource theory e good management theory. De acordo com a slack resource theory, quando há uma folga de recursos financeiros a empresa passa a investir em responsabilidade social, ou seja, primeiro há um bom desempenho financeiro para consequentemente ocorrer o bom desempenho social, então a variável CSP é considerada como dependente, e a CFP como variável explicativa. De acordo com a good management theory, primeiro ocorre um bom desempenho social que reflete numa boa gestão de stakeholders. O bom relacionamento com os stakeholders resultaria em funcionários mais satisfeitos, clientes mais fiéis, fornecedores parceiros, comunidade engajada, boa imagem da empresa, entre outros aspectos que causariam um bom desempenho financeiro. Nesta perspectiva, primeiro há uma boa CSP para consequentemente ocorrer uma boa CFP, ou seja, a CSP é considerada variável explicativa e a CFP é variável dependente. Nas revisões de Margolis e Walsh (2003), Orlitzky, Schmidt e Rynes (2003) e Boaventura, Silva e Bandeira de Mello (2012), a maioria dos estudos apoia-se na good management theory.

Esta pesquisa também se apoia na good management theory ao defender que a adoção de práticas socialmente responsáveis melhora o desempenho financeiro da empresa (CHENG, IOANNOU; SERAFEIM, 2014; MCWILLIAMS; SIEGEL, 2011), ou seja, o aumento do desempenho social pode ser entendido como um aumento da satisfação dos stakeholders, o que gera vantagens competitivas e ganhos financeiros para empresa. Em função disso, sugere-se testar em empresas brasileiras a seguinte hipótese: 
H1: A CSP tem um impacto positivo na CFP.

\subsection{Recursos intangíveis como mediadores da relação CSP-CFP}

Apesar de haver na literatura um predomínio de estudos que encontraram que a CSP gera valor financeiro a empresa por meio de aumento do valor da ação, diminuição do risco e de custos, maior atratividade de investidores e outros (MARGOLIS; WALSH, 2003; ORLITZKY; SCHMIDT; RYNES, 2003), é muito difícil provar uma relação causal direta entre CSP e CFP (LUO et al., 2015; MADORRAN; GARCIA, 2016). Muitos fatores endógenos podem influenciar a adoção de práticas de CSP, por exemplo, relacionados a recursos intangíveis. Esses fatores influenciam tanto a CSP como a CFP, o que poderia confundir os resultados. Além disso, a própria CFP pode influenciar a adoção de práticas de CSP, invertendose a direção da relação causal (NELLING; WEBB, 2009; WADDOCK; GRAVES, 1997). Por esse motivo, o presente trabalho buscou testar se existe uma correlação entre CSP e CFP com a mediação dos recursos intangíveis da empresa.

Os recursos intangíveis da empresa, incluindo inovação, capital humano, reputação e cultura organizacional são variáveis mediadoras entre CSP e CFP (SURROCA; TRIBÓ; WADDOCK, 2010). Ou seja, a CSP terá um efeito positivo no desempenho financeiro da empresa à medida que possibilite o desenvolvimento de recursos intangíveis, caso contrário poderá ter efeitos neutros e até mesmo negativos (HILLMAN; KEIM, 2001; SURROCA; TRIBÓ; WADDOCK, 2010).

A CSP pode levar ao desenvolvimento de ativos intangíveis por meio de diversos mecanismos. Por exemplo, as políticas ambientais e de responsabilidade social podem aumentar a capacidade de a empresa inovar, tanto em termos de produtos como de processos. Essa inovação aumentaria a qualidade e atratividade de seus produtos resultando numa melhor CFP (SURROCA; TRIBÓ; WADDOCK, 2010). Isso ocorre, pois empresas dispostas a promover relações com stakeholders que vão além das exigências legais conseguem estimular novas percepções sobre atividades, processos e desenvolvimento de novos produtos (HART; DOWELL, 2011; ORSATO et al., 2015). A empresa pode interagir com múltiplos stakeholders para identificar novas abordagens que proporcionam benefícios tanto para o negócio quanto para sociedade (MARTINUZZI; KRUMAY, 2013). 
A CSP também melhora a acumulação de capital humano uma vez que as empresas percebidas como responsáveis tendem a atrair e reter melhores candidatos a emprego e influenciar positivamente na motivação dos funcionários. Estudos como de Backhaus, Stone e Heiner (2002) exploraram a relação entre CSP e atratividade de funcionários e mostraram que pessoas que procuram emprego analisam as atividades da empresa relacionadas a meio ambiente, relação com a comunidade, relação com trabalhadores e diversidade, para escolherem onde trabalhar. Além disso, empresas com práticas e políticas de responsabilidade social desenvolvidas possuem maiores chances de conseguir um bom clima organizacional (BRANCO; RODRIGUES, 2006) e de reter os melhores talentos (BARRENA-MARTÍNEZ et al., 2015). A acumulação de capital humano reduziria custos e pode ser uma fonte de vantagem competitiva (SURROCA; TRIBÓ; WADDOCK, 2010).

A CSP pode ser, também, um indicativo de que a empresa adota práticas aderentes com as expectativas dos stakeholders, o que consequentemente aumenta a reputação da empresa. A reputação faz parte dos ativos intangíveis da empresa e é construída e mantida pela capacidade da empresa em atender as expectativas dos múltiplos stakeholders (MAHON; WARTICK, 2012). Além disso, estudos empíricos demonstraram que existe uma relação positiva entre reputação e desempenho financeiro (e. g. RAITHEL; SCHWAIGER, 2015; ROBERTS; DOWLING, 2002) e que ações ligadas à CSP melhoram a reputação da empresa (e. g. GODFREY, 2005; TURBAN; GREENING, 1997). Neste sentido, a construção de uma reputação positiva assegura a participação dos stakeholders em continuar contribuindo com as atividades corporativas, que é fundamental para a sobrevivência e lucratividade contínua da empresa. Por fim, a adoção de práticas de responsabilidade social contribui para mudanças na cultura organizacional. Os stakeholders internos da empresa podem perceber as iniciativas voltadas a stakeholders externos - como proteção ao meio ambiente, investimento em comunidades locais e filantropia- como sinais de justiça e equidade organizacional (AGUILERA et al., 2007). A percepção de justiça e equidade decorrente da CSP pode afetar a identificação do stakeholder interno com os valores e a imagem organizacional (ONKILA; 2015), podendo levar a uma identificação favorável com todas as outras atividades da empresa (BRUNTON; EWEJE; TASKIN, 2015). Como resultado, promove-se maior comprometimento dos stakeholders internos (COLLIER; ESTEBAN, 2007), impactando positivamente no desempenho financeiro (HUSELID, 1995).

Em função do exposto, sugere-se testar em empresas brasileiras, a seguinte hipótese:

H2: Recursos intangíveis são mediadores da relação entre CSP e CFP. 


\section{METODOLOGIA}

Esta pesquisa tem como objetivo testar se os recursos intangíveis são mediadores da relação entre CSP e CFP. Para isso, foram testadas duas hipóteses em uma amostra de empresas brasileiras. A Figura 1 apresenta o modelo testado nesta pesquisa.

Figura 1 - Modelo da pesquisa

$\mathrm{H} 2$

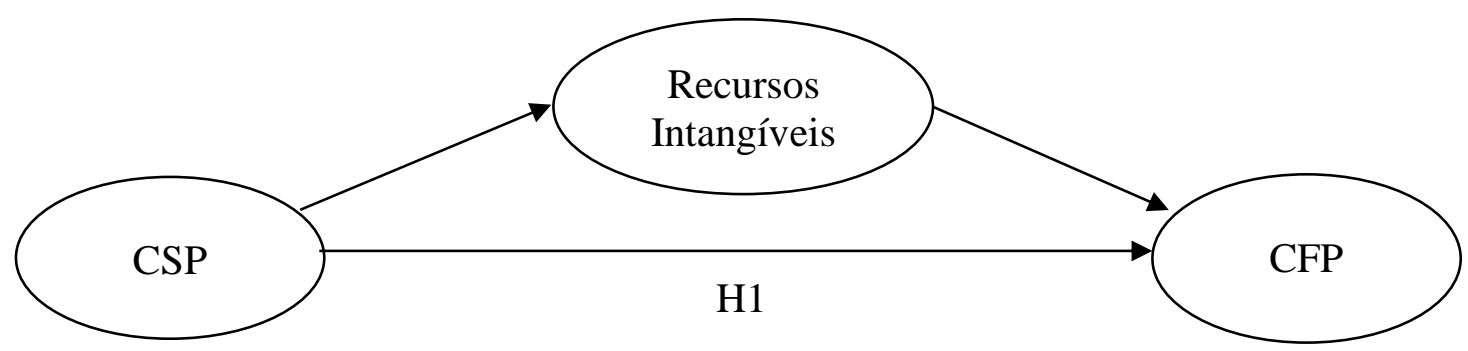

Fonte: elaboração própria.

As equações testadas, referentes a cada hipótese discutida no referencial teórico, são:

$$
\begin{aligned}
& \mathrm{CFP}=\beta 0+\mathrm{CSP} \beta 1+\text { tamanho } \beta 2+\operatorname{setor} \beta 3 \\
& \mathrm{CFP}=\beta 0+\mathrm{CSP} \beta 1+\text { intangíveis } \beta 2+\text { tamanho } \beta 3+\text { setor } \beta 4 \quad \text { (Modelo 2) }
\end{aligned}
$$

\subsection{Universo e amostra}

O universo da pesquisa compreende 368 empresas listadas na bolsa de valores, mercadorias e futuros de São Paulo - Bolsa, Brasil, Balcão (B3). Para seleção da amostra, procedeu-se a organização e tratamento da base de dados. A base Economatica lista ações ordinárias e preferenciais, apresentando, portanto, mais de uma observação por empresa. Mitigando ruídos na análise de dados, retirou-se as duplicidades, considerando-se apenas uma observação por empresa sob o critério de liquidez por ativo de negociação. Retirou-se também, do banco de dados desta pesquisa, as empresas que apresentavam missing value em pelo menos uma das variáveis de interesse ou de controle. A amostra total analisada foi de 230 empresas com ações negociadas na Bolsa, Brasil, Balcão (B3) em 2013. Os dados sobre o desempenho financeiro das empresas, assim como dados referentes às características das empresas tamanho e setor - foram coletados do banco de dados da Economatica. 


\subsection{Mensuração das variáveis}

\subsubsection{Mensuração da CSP - Variável explicativa}

Para mensurar a CSP, Waddock e Graves (1997) afirmam que as pesquisas sobre o tema usam medidas de percepção e comportamento / survey; índices reputacionais; estudos de caso; análise de conteúdo de documentos e social disclosure. De forma semelhante, Orlitzky, Schmidt e Rynes (2003) destacam quatro estratégias de mensuração do constructo CSP: disclosure; avaliações reputacionais; auditorias sociais, processos e resultados observáveis; e princípios e valores gerenciais.

Nesta pesquisa, a variável CSP é decorrente da participação ou não da empresa no Índice de Sustentabilidade Empresarial (ISE). O ISE é considerado uma ferramenta para análise comparativa do desempenho das empresas listadas na bolsa de valores, mercadorias e futuros de São Paulo - Bolsa, Brasil, Balcão (B3) sob o aspecto da sustentabilidade corporativa, baseada em eficiência econômica, equilíbrio ambiental, justiça social e governança corporativa (GVces, 2014). O ISE foi criado com o objetivo de criar uma carteira de ações que fosse um referencial para os investimentos socialmente responsáveis e estimular a responsabilidade ética das empresas (GVces, 2014). O questionário que avalia se as atividades das empresas são consideradas sustentáveis é composto por sete dimensões: ambiental, econômico-financeira, geral, governança corporativa, mudanças climáticas, natureza do produto e social (GVces, 2014).

O índice tem sido utilizado em diversas pesquisas para mensurar o desempenho social das empresas. Pesquisas anteriores utilizaram o ISE como uma variável binária (e. g. GOLDNER NUNES et al., 2010; MELO; ALMEIDA; SANTANA, 2012; TEIXEIRA; NOSSA; FUNCHAL, 2011). No contexto internacional, também se observa o uso de variáveis binárias para análise da CSP, como no estudo de Chan, Chou e Lo (2017). Em consistência com esses estudos anteriores, caso a empresa estivesse listada no índice atribuiu-se 1 e caso não estivesse atribuiu-se $0 . \mathrm{Na}$ amostra analisada nesta pesquisa, 26 empresas receberam valor "1" para a CSP e as demais empresas listadas receberam valor " 0 ”. Esse resultado é após a retirada dos missing values. 


\subsubsection{Mensuração de recursos intangíveis - Variável mediadora}

O grau de intangibilidade dos recursos da empresa, ou intangibilidade dos recursos, refere-se ao valor dos ativos intangíveis da empresa com relação a seus recursos tangíveis. $\mathrm{O}$ valor dos ativos tangíveis da empresa é o custo de substituição desses ativos (custo atual de compra de um ativo com capacidade produtiva equivalente) e pode ser estimado apropriadamente ajustando dados contábeis. O valor dos recursos intangíveis da empresa pode ser estimado como a diferença entre o valor de mercado da empresa e os custos de substituição de seus ativos tangíveis (ANDERSEN, 1992).

Seguindo o mesmo procedimento de pesquisas anteriores, no presente estudo, foi utilizado o Market-to-book para medir a intangibilidade dos recursos (JOIA, 2000; KAYO et al., 2006). O Market-to-book é obtido pela razão entre o valor de mercado e o valor contábil da empresa. Os ativos tangíveis são capitalizados, ou seja, reconhecidos como ativos e informados no balanço das empresas. Porém, os intangíveis são debitados da demonstração de resultados juntamente com as despesas regulares, como salários, aluguéis e juros. Como resultado, o valor contábil dos ativos não reflete o estoque de ativos intangíveis que resulta de investimento acumulado, mas o valor de mercado sim. Joia (2000) baseia-se em diversos autores ao considerar que o valor de mercado é formado pelo book value (valor contábil) e pelo capital intelectual (intangíveis). Portanto, o autor considera que o Market-to-book é uma proxy para recursos intangíveis.

\subsubsection{Mensuração da CFP - Variável dependente}

Para mensurar a CFP, foi utilizada a variável ROA (Return on Assets) com o objetivo de aferir comparabilidade ao estudo (BRITO; BRITO, 2012). Segundo Boaventura, Silva e Bandeira de Mello (2012), que analisaram a publicação acadêmica sobre a relação CSP-CFP no período de 1996 a 2010, o indicador ROA é a variável mais utilizada nos estudos para mensuração da CFP, pois é uma proxy que visa medir o retorno sobre o investimento realizado pela empresa. A variável ROA, extraída da Economatica para cada empresa no período analisado, pode ser obtido através da razão entre o resultado líquido e o ativo total (ASSAF NETO, 2002). 


\subsubsection{Mensuração das variáveis de controle}

Uma vez que outras variáveis podem influenciar o desempenho da empresa, utilizou-se como variáveis de controle: 1) tamanho da empresa, medido pelo logaritmo natural do ativo total (KAYO, 2015); 2) setor de atuação, classificado pelo critério utilizado na Economatica. Essas duas variáveis de controle foram escolhidas, pois são amplamente aceitas e utilizadas nos estudos de CSP-CFP (BOAVENTURA; SILVA; BANDEIRA DE MELLO, 2012; MCWILLIAMS; SIEGEL, 2000).

\subsection{Análise de dados}

A Hipótese 1 foi estimada por meio de regressão múltipla pelo Método dos Quadrados Mínimos (MQO). Para evitar viés nos estimadores, a técnica de regressão por MQO exige o atendimento de algumas premissas. Uma das premissas da técnica refere-se a homocedasticidade dos resíduos, a qual foi testada por meio do teste de Breusch-Pagan. Os modelos que não atenderam a essa premissa foram estimados com erros robustos para heterocedasticidade.

A técnica de regressão também é sensível à presença de multicolinearidade e de outliers. A multicolinearidade foi verificada por meio do fator de inflação de variação (variance inflation factor - VIF), que não deve ultrapassar o valor de 10 (HAIR et al., 2009). Para análise de outliers influentes foi empregada a técnica DFITS que é um indicador global que avalia a influência de outlier nos coeficientes de regressão como um todo (AGUINIS; GOTTFREDSON; JOO, 2013). Como critério para identificar se há ou não a presença de outliers influentes, utilizou-se como ponto de corte valores Dfits maiores que 1. Aguinis, Gottfredson e Joo (2013) sugerem que, quando identificada a presença de outliers influentes, os pesquisadores apresentem os resultados da regressão com a amostra completa e com a exclusão das observações influentes. Em função disso, os resultados são apresentados considerando a amostra completa e a amostra com a exclusão dos outliers.

A Hipótese 1 deste estudo foi testada por meio da seguinte equação:

$$
\mathrm{CFP}=\beta 0+\operatorname{CSP} \beta 1+\text { tamanho } \beta 2+\text { setor } \beta 3 \quad \text { (Modelo } 1)
$$


Para testar a Hipótese 2, de mediação, empregou-se o método descrito por Baron e Kenny (1986), o qual consiste na estimação de três modelos de regressão.

Regressões:

$\mathrm{a}=\mathrm{CSP}$ como regressor de Market-to-book

$\mathrm{b}=$ Market-to-book como regressor de CFP

$\mathrm{c}=$ CSR como regressor de CFP

c'= CSR como variável independente e Market-to-book como variável mediadora sobre

CFP

Os modelos foram testados segundo as seguintes equações:

\begin{tabular}{|l|l|l|}
\hline Regressões & Equações com controles & Modelos \\
\hline $\mathrm{a}$ & Market-to-book $=\beta 0+\mathrm{CSP} \beta 1+\operatorname{tamanho} \beta 2+$ setor $\beta 3$ & Modelo 2 \\
\hline $\mathrm{b}$ & $\mathrm{CFP}=\beta 0+$ Market-to-book $\beta 1+\operatorname{tamanho} \beta 2+$ setor $\beta 3$ & Modelo 3 \\
\hline $\mathrm{c}$ & $\mathrm{CFP}=\beta 0+\mathrm{CSP} \beta 1+\operatorname{tamanho} \beta 2+\operatorname{setor} \beta 3$ & Modelo 1 \\
\hline $\mathrm{c}^{\prime}$ & $\mathrm{CFP}=\beta 0+\mathrm{CSP} \beta 1+$ Market-to-book $\beta 2+\operatorname{tamanho} \beta 3+\operatorname{setor} \beta 4$ & Modelo 4 \\
\hline
\end{tabular}

Segundo Baron e Kenny (1986), uma variável funciona como um mediador quando são atendidas três condições: (a) a variável independente está significativamente associada com o mediador (b) o mediador afeta significativamente a variável dependente e (c) a variável independente não mais apresenta uma relação significativa na presença do mediador. Para testar a significância do efeito da variável independente sobre a variável dependente por meio do mediador foi utilizado o teste de Sobel (BARON; KENNY, 1986). Complementou-se as análises com o teste RESET de Ramsey (1969) que verifica a presença de variáveis omitidas no modelo. A hipótese nula do teste foi rejeitada, o que indica a presença de variáveis omitidas ou questões de especificação no modelo. No entanto, considera-se que a relação estudada é complexa e envolve diferentes tipos de variáveis, que não estão no foco deste estudo. Portanto, indica-se que é uma limitação do método que ocorre em diferentes estudos da área de gestão (e. g. BRITO; OLIVEIRA, 2016; VIEIRA; KREUTZ, 2018).

\section{RESULTADOS}

A amostra total analisada foi de 230 empresas listadas em 2013 com ações negociadas na bolsa de valores, mercadorias e futuros de São Paulo - Bolsa, Brasil, Balcão (B3). Destas 230 empresas, 37 empresas participaram da carteira ISE no a. A Tabela 1 mostra a distribuição das empresas pelos 21 setores, segundo o critério de classificação fornecido pela base Economatica. 
Tabela 1 - Relação de empresas por setor de atuação

\begin{tabular}{l|l|l|l}
\hline Setor & Freq. & Percent. & Cum. \\
\hline Energia & 30 & 13,04 & 13,04 \\
\hline Construção & 19 & 8,26 & 21,3 \\
\hline Siderurgia & 19 & 8,26 & 29,56 \\
\hline Têxtil & 18 & 7,83 & 37,39 \\
\hline Comércio & 16 & 6,96 & 44,35 \\
\hline Serviços & 16 & 6,96 & 51,31 \\
\hline Administração & 14 & 6,09 & 57,4 \\
\hline Alimentos & 14 & 6,09 & 63,49 \\
\hline Indústrias & 11 & 4,78 & 68,27 \\
\hline Química & 10 & 4,35 & 72,62 \\
\hline Veículos & 10 & 4,35 & 76,97 \\
\hline Transporte & 9 & 3,91 & 80,88 \\
\hline Eletroeletrônicos & 7 & 3,04 & 83,92 \\
\hline Locadora de imóveis & 7 & 3,04 & 86,96 \\
\hline Minerais & 6 & 2,61 & 89,57 \\
\hline Máquinas & 5 & 2,17 & 91,74 \\
\hline Telecomunicações & 5 & 2,17 & 93,91 \\
\hline Finanças & 4 & 1,74 & 95,65 \\
\hline Papel & 4 & 1,74 & 97,39 \\
\hline Petróleo & 3 & 1,3 & 98,69 \\
\hline Água e Saneamento & 3 & 1,3 & 99,99 \\
\hline Total & 230 & & 100 \\
\hline Fote & & &
\end{tabular}

Fonte: elaboração própria.

A maior concentração de empresas está no setor de energia com 30 empresas, equivalente a 13,04\% do total da amostra. A segunda maior frequência foi dos setores de construção e siderurgia, com 19 empresas cada $(8,26 \%)$, seguidos do setor têxtil com 18 empresas. Os setores com menor número de empresas na amostra foram os setores de petróleo e água e esgoto, com 3 empresas cada. Apesar de o setor de energia apresentar a maior frequência, destaca-se que a amostra é composta por setores diversificados, sem que haja a predominância de um setor.

A Tabela 2 apresenta as estatísticas descritivas para todas as variáveis métricas utilizadas neste estudo.

Tabela 2 - Estatísticas descritivas

\begin{tabular}{l|r|r|r|r|r}
\hline Variável & Obs. & \multicolumn{1}{l|}{ Média } & Desv. Pad. & Mín. & \multicolumn{1}{l}{ Máx. } \\
\hline ROA & 230 & 1,85 & 10,02 & $-51,80$ & 33,90 \\
\hline Market-to-book & 230 & 742,20 & 945,93 & 12,75 & 8083,42 \\
\hline Tamanho & 230 & 14,69 & 1,91 & 6,65 & 20,44 \\
\hline
\end{tabular}

Fonte: elaboração própria. 
O ROA médio foi de 1,85 com um desvio-padrão de 10,02. O tamanho médio das empresas foi de 14,69 com desvio-padrão de 1,91. O Market-to-book médio foi de 742,2 com desvio-padrão de 945,93. Estes resultados indicam alta variabilidade no ROA, Market-to-book e tamanho das empresas da amostra.

A Tabela 3 apresenta a matriz de correlação entre as variáveis.

Tabela 3 - Matriz de correlação

\begin{tabular}{lllll}
\hline & ROA & CSP & Market-to-book & Tamanho \\
\hline ROA & 1 & & & \\
CSP & $0,1252^{*}$ & 1 & & \\
Market-to-book & $0,3300^{*}$ & 0,029 & 1 & 1 \\
\hline Tamanho & $0.2087^{*}$ & $0.4304^{*}$ & $-0.1484 *$ & \\
***Significante a p $<0.01 ; * *$ Significante a p $<0.05 ; *$ Significante a $<0.10$. & \\
Fonte: elaboração própria.
\end{tabular}

As variáveis CSP e Market-to-book apresentaram uma relação positiva e significante com a variável dependente ROA. A variável tamanho, embora positiva, não foi significante com ROA. Por outro lado, está positiva e significantemente correlacionada com CSP, indicando que quanto maior a empresa, maior é o desempenho social. Esses resultados são normalmente encontrados em pesquisas da área, porém devem ser observados com cuidado uma vez que as correlações não consideram a influência de outras variáveis.

\subsection{Teste da Hipótese 1}

Nas Tabelas 4 e 5, são apresentados os resultados decorrentes das regressões que testaram as hipóteses propostas neste estudo. Ressalta-se que as tabelas apresentam os modelos estimados com e sem a presença de outliers influentes, conforme sugerido por Aguinis, Gottfredson e Joo (2013). Os modelos que apresentaram resíduos homocedásticos foram estimados com erros robustos para heterocedasticidade. Quanto ao VIF, nenhum dos modelos apresentou evidências de multicolinearidade uma vez que o VIF máximo foi igual a 2,98.

O Modelo 1, apresentado na Tabela 4, testa a relação entre CSP e CFP na presença das variáveis de controle tamanho e setor, porém sem a presença da variável mediadora Market-tobook. Devido à grande quantidade de variáveis dummy para controle do setor, estas foram suprimidas das tabelas. 
Tabela 4 - Resultados da análise de regressão

\begin{tabular}{lcc}
\hline Modelo 1 - Var dep. ROA. & & \\
\hline CSP & Amostra Completa & Sem obs. inf. \\
Market-to-book & $3,048^{*}$ & $2,751^{*}$ \\
& $(1,630)$ & $(1,576)$ \\
Tamanho & - & - \\
& & \\
Setor & $0,995^{* *}$ & $0,843^{*}$ \\
Constante & $(0,451)$ & $(0,438)$ \\
& Sim & Sim \\
\hline VIF Max & $-13,564^{* *}$ & $-11,480^{*}$ \\
VIF Médio & $(6,608)$ & $(6,505)$ \\
Homocedasticidade & 2,95 & 2,95 \\
R & 1,69 & 1,68 \\
F & Não & Não \\
\hline Obs. & 0,1259 & 0,1278 \\
\end{tabular}

Nota: os valores apresentados na primeira linha de cada variável são os coeficientes. Na segunda linha, entre parênteses, está o erro padrão. ***Significante a p < 0.01; **Significante a p < 0.05; *Significante a $<0.10$. Fonte: elaboração própria.

Observa-se que a CSP apresenta uma relação positiva e significante com CFP, ao nível de $10 \%$, nas duas amostras. O beta da amostra completa foi igual a 3,048 e da amostra sem a presença de observações influentes foi de 2,715. A estatística F indica que os modelos, como um todo, foram estatisticamente significantes a $5 \%$ para explicar a variação de ROA, sendo $\mathrm{R}^{2}$ igual a $12,59 \%$ para a amostra completa e $12,78 \%$ para a amostra sem outliers. Esse resultado suporta a Hipótese 1, de que a CSP está positivamente associada à CFP.

\subsection{Teste da Hipótese 2}

A Tabela 5 apresenta as regressões utilizadas para análise da relação de mediação da variável Market-to-book, utilizada como proxy para recursos intangíveis. Conforme descrito na seção de metodologia, o método de Baron e Kenny (1986) prevê a estimação de três modelos de regressão: (a) CSP como regressor de Market-to-book; (b) Market-to-book como regressor de CFP, (c) CSP como regressor de CFP e (c') CSP como variável independente e Market-tobook como variável mediadora sobre CFP. Uma vez que a regressão "c" foi utilizada para testar a Hipótese 1, conforme apresentado na Tabela 4 (Modelo 1), a Tabela 5 apresenta apenas as regressões a (Modelo 2), b (Modelo 3) e c' (Modelo 4). 
RECURSOS INTANGÍVEIS E A RELAÇÃO ENTRE DESEMPENHO SOCIAL E DESEMPENHO FINANCEIRO EM EMPRESAS BRASILEIRAS

Tabela 5 - Resultados da análise de mediação

\begin{tabular}{|c|c|c|c|c|c|c|}
\hline & \multicolumn{2}{|c|}{$\begin{array}{c}\text { Modelo 2 - Var dep. } \\
\text { Market-to-book }\end{array}$} & \multicolumn{2}{|c|}{$\begin{array}{c}\text { Modelo 3 - Var dep. } \\
\text { ROA }\end{array}$} & \multicolumn{2}{|c|}{$\begin{array}{c}\text { Modelo 4 - Var dep. } \\
\text { ROA }\end{array}$} \\
\hline & $\begin{array}{l}\text { Amostra } \\
\text { Completa }\end{array}$ & $\begin{array}{l}\text { Sem obs. } \\
\text { inf. }\end{array}$ & $\begin{array}{l}\text { Amostra } \\
\text { Completa }\end{array}$ & \begin{tabular}{|l}
$\begin{array}{l}\text { Sem obs. } \\
\text { inf. }\end{array}$ \\
\end{tabular} & $\begin{array}{l}\text { Amostra } \\
\text { Completa }\end{array}$ & $\begin{array}{l}\text { Sem obs. } \\
\text { inf. }\end{array}$ \\
\hline CSP & $\begin{array}{l}453,39 * \\
(247,89)\end{array}$ & $\begin{array}{l}380,47 * * \\
(155,79)\end{array}$ & - & - & \begin{tabular}{|l|}
1,387 \\
$(1,436)$
\end{tabular} & $\begin{array}{l}1,792 \\
(1,211)\end{array}$ \\
\hline $\begin{array}{l}\text { Market-to- } \\
\text { book }\end{array}$ & - & & $0,004 * * *$ & $0,005 * * *$ & $0,004 * * *$ & $0,006 * * *$ \\
\hline Tamanho & $\begin{array}{l}-81,799 \\
(95,504) \\
\end{array}$ & $\begin{array}{l}-28,234 \\
(33,083) \\
\end{array}$ & $\begin{array}{l}(0,0014) \\
1,385 * * * \\
(0,504) \\
\end{array}$ & \begin{tabular}{|l|}
$(0,0007)$ \\
0,638 \\
$(0,390)$ \\
\end{tabular} & $\begin{array}{l}(0,001) \\
1,284 * * \\
(0,545) \\
\end{array}$ & $\begin{array}{l}(0,0007) \\
0,516 \\
(0,416) \\
\end{array}$ \\
\hline Setor & Sim & Sim & Sim & Sim & Sim & Sim \\
\hline \multirow[t]{2}{*}{ Constante } & 2263,1 & $1011,1 * *$ & $-23,16 * * *$ & $-11,09 *$ & $-21,85^{* * *}$ & $-9,312$ \\
\hline & $(1765,0)$ & $(483,21)$ & $(7,852)$ & $(5,704)$ & $(8,445)$ & $(6,079)$ \\
\hline VIF Max & 2,95 & 3,03 & 2,95 & 2,99 & 2,98 & 3,03 \\
\hline VIF Médio & 1,69 & 1,73 & 1,67 & 1,70 & 1,68 & 1,70 \\
\hline Homoce & Não & Não & Não & Não & Não & Não \\
\hline $\mathrm{R}^{2}$ & 0,1217 & 0,1517 & 0,2296 & 0,3482 & 0,2310 & 0,3514 \\
\hline $\mathrm{F}$ & $1,87 * *$ & $1,85^{* *}$ & $4,34 * * *$ & 7,36 *** & $3,88 * * *$ & $6,36^{* * *}$ \\
\hline Obs. & 230 & 226 & 230 & 225 & 230 & 225 \\
\hline
\end{tabular}

Nota: os valores apresentados na primeira linha de cada variável são os coeficientes. Na segunda linha, entre parênteses, está o erro padrão. ***Significante a $\mathrm{p}<0.01 ; * *$ Significante a $\mathrm{p}<0.05 ; *$ Significante a $<0.10$. Fonte: elaboração própria.

No modelo 2a, foi testada a relação entre CSP e Market-to-book. Neste modelo, obtevese uma relação positiva e significante entre CSP e Market-to-book $(\beta=453,39 ; \mathrm{p}<0,10)$. Na amostra com a retirada dos outliers influentes, a CSP apresenta beta igual a 380,47, porém com maior significância estatística $(\mathrm{p}<0,05)$. O Modelo $2 \mathrm{~b}$ testa a relação entre a variável mediadora e ROA. Observa-se que o Market-to-book afeta de forma positiva e significante o ROA ( $\beta=$ 0.004; $\mathrm{p}<0,01)$. Neste modelo, a exclusão dos outliers não altera a relação positiva e significante $(\beta=0.005 ; \mathrm{p}<0,01)$.

No Modelo 2c', as variáveis CSP e Market-to-book são incluídas como regressores no mesmo modelo. Com a inclusão de Market-to-book, observa-se que a relação positiva e significante de CSP e CFP, apresentada na Tabela 4 - Modelo 1, perde significância estatística de $\beta=3,048$ e $p<0,10$ para $\beta=1,387$ e $p>0,10$, na amostra completa e $\beta=2,715$ e $p<0,10$ para $\beta=1,792$ e p>0,10 na amostra sem outliers. Essa mudança indica que há uma relação de mediação total nas duas amostras. Em termos de explicação da variação de ROA, o Modelo 2c' é o modelo com maior $\mathrm{R}^{2}, 23,10 \%$ para a amostra completa e 35,14 para a amostra sem observações influentes. 
Por fim, os resultados do teste de Sobel para a amostra sem outliers foram diferentes de zero $(Z=2,32 ; p<0,05)$, indicando que o efeito indireto foi estatisticamente significante. Assim, estes resultados indicam que os recursos intangíveis mediam a relação entre CSP e CFP, fornecendo suporte para a Hipótese 2.

\section{DISCUSSÃO E CONCLUSÕES}

O presente estudo teve como objetivo testar a relação entre CSP e CFP em empresas brasileiras, assim como a mediação dos recursos intangíveis nessa relação. A hipótese de que há uma correlação positiva e significante entre CSP e CFP foi confirmada. Este resultado corrobora com os achados de outros pesquisadores, tanto em pesquisas com amostras de empresas de países desenvolvidos (GRIFFIN; MAHON, 1997; MARGOLIS; WALSH, 2003; ORLITZKY; SCHMIDT; RYNES, 2003), quanto em pesquisas que consideraram o contexto brasileiro, a exemplo dos estudos de Abreu et al. (2009), Beato, Souza e Parisotto (2009) e Orellano e Quiota (2011). Embora a relação direta entre CSP e CFP seja positiva e significante, os resultados indicam uma fraca associação entre as variáveis, uma vez que a significância estatística de CSP se deu apenas a $10 \%$ e o $\mathrm{R}^{2}$ do modelo apresentou uma baixa explicação da variação de ROA, apenas $12 \%$. Este resultado reforça a ideia defendida pelo presente estudo de que os modelos que testam a relação direta entre CSP e CFP apresentam relações espúrias de causalidade (RUF et al., 2001; SURROCA; TRIBÓ; WADDOCK, 2010; LUO et al., 2015).

Contribuindo com esta questão, a segunda hipótese deste estudo considera que a relação CSP-CFP não é uma relação direta, sendo mediada pelos recursos intangíveis. Os resultados confirmaram esta hipótese, indicando que CSP afeta os recursos intangíveis da organização, tais como inovação, capital humano, reputação e cultura, os quais, por sua vez, afetam a CFP. O resultado encontrado na amostra brasileira está alinhado aos resultados de Surroca, Tribó e Waddock (2010), que testaram esta relação com dados de 599 empresas da base Sustainalytics.

A mediação dos recursos intangíveis na relação CSP-CFP evidencia que o investimento em ações e práticas de responsabilidade social não garante à empresa ganhos financeiros e vantagem competitiva. A CSP deve promover o desenvolvimento de recursos intangíveis, os quais são valiosos, raros e difíceis de serem adquiridos e copiados (BARNEY, 1991; DIERICKX; COOL, 1989; WERNERFELT, 1984) e, por meio deles, obter maior CFP e vantagem competitiva. Dessa forma, torna-se importante que os estudos em CSP busquem 
analisar não somente se a CSP leva a CFP, mas sim entender quais as condições que levam a CSP a criar valor para empresa. Essas condições podem estar relacionadas, por exemplo, a projetos sociais desenhados estrategicamente com o objetivo de gerar vantagem competitiva (HUSTED; ALLEN; KOCK, 2015) ou a ações sociais que sejam relacionadas a stakeholders primários, como funcionários, clientes, fornecedores e comunidade (HILLMAN; KEIM, 2001).

O presente estudo contribui com a literatura de CSP testando empiricamente a mediação dos recursos intangíveis na relação CSP-CFP em empresas brasileiras, uma vez que a maioria dos estudos existentes sobre esta relação está concentrada em empresas norte-americanas e europeias. No Brasil, existem estudos que buscaram testar essa relação, porém, estão claramente em defasagem com relação à quantidade de evidências empíricas e, principalmente, com relação aos procedimentos metodológicos empregados, quando comparados aos estudos internacionais.

Neste sentido, embora muitos estudos tenham tratado deste tema, o avanço na área ainda enfrenta desafios. No presente estudo, apesar de suas contribuições, essas limitações também estão presentes. A primeira delas refere-se à mensuração da CSP, a qual foi feita por meio de uma variável binária. Estudos futuros podem buscar diferentes variáveis ou proxies para captar a CSP das empresas. A segunda limitação refere-se à endogeneidade existente na relação entre CSP-CFP. A endogeneidade existe quando a variável preditora influência na predita e viceversa, ou seja, há evidências de que a CSP influencie a CFP, porém a CFP também pode influenciar a CSP, uma vez que um maior desempenho financeiro resulta em mais recursos financeiros disponíveis para serem investidos em ações de responsabilidade social.

Futuras pesquisas podem investigar a mediação dos recursos intangíveis na relação CSP-CFP em outros contextos e em outros recortes temporais. Além disso, a investigação de outras variáveis que possam afetar a relação CSP-CFP, tanto em termos de mediação quanto de moderação, são estudos importantes para a evolução desta teoria.

\section{REFERÊNCIAS}

ABREU, M. C. S. de; CASTRO JUNIOR, O. V. de; SOARES, F. de A.; SILVA FILHO, J. C. L. da. Efeito da Conduta Social sobre a Performance Econômica: Evidências da Indústria Têxtil Brasileira. Contabilidade Vista \& Revista, v. 20, n. 1, p. 119-142, 2009.

ABREU, M. C. S. de; CUNHA, L. T. da; BARLOW, C. Y. Institutional dynamics and organizations affecting the adoption of sustainable development in the United Kingdom and 
Brazil. Business Ethics: A European Review, v. 24, n. 1, p. 73-90, 2015. <https://doi.org/10.1111/beer.12074>

AGUILERA, R. V.; RUPP, D. E.; WILLIAMS, C. A.; GANAPATHI, J. Putting the S back in corporate social responsibility: A multilevel theory of social change in organizations. Academy $\begin{array}{llllllll}\text { of Management Review, } & \text { v. } 32, \quad \text { n. } 3, \quad \text { p. } & 836-863,\end{array}$ <https://doi.org/10.5465/amr.2007.25275678>

AGUINIS, H.; GLAVAS, A. What we know and don't know about corporate social responsibility a review and research agenda. Journal of Management, v. 38, n. 4, p. 932-968, 2012. <https://doi.org/10.1177/0149206311436079>

AGUINIS, H.; GOTTFREDSON, R. K.; JOO, H. Best-practice recommendations for defining, identifying, and handling outliers. Organizational Research Methods, v. 16, n. 2, p. 270-301, 2013. <https://doi.org/10.1177/1094428112470848>

ANDERSEN, A. The valuation of intangible assets. London: Economist Intelligence Unit, 1992.

ASSAF NETO, A. Estrutura e análise de balanços: um enfoque econômico-financeiro. 7. ed. São Paulo: Atlas, 2002.

BACKHAUS, K. B.; STONE, B. A.; HEINER, K. Exploring The relationship between corporate social performance and employer attractiveness. Business \& Society, v. 41, n. 3, p. 292-318, 2002. <https://doi.org/10.1177/0007650302041003003>

BARNETT, M. L. Stakeholder influence capacity and the variability of financial returns to corporate social responsibility. Academy of Management Review, v. 32, n. 3, p. 794-816, 2007. <https://doi.org/10.5465/amr.2007.25275520>

BARNETT, M. L.; SALOMON, R. M. Does it pay to be really good? Addressing the shape of the relationship between social and financial performance. Strategic Management Journal, v. 33, n. 11, p. 1304-1320, 2012. 〈https://doi.org/10.1002/smj.1980>

BARNEY, J. B. Firm Resources and Sustained Competitive Advantage. Journal of Management, v. 17, n. 1, 1991, p. 99-120. <https://doi.org/10.1177/014920639101700108>

BARON, R. M.; KENNY, D. A. The moderator-mediator variable distinction in social psychological research: Conceptual, strategic, and statistical considerations. Journal of Personality and Social Psychology, v. 51, n. 6, p. 1173, 1986. <https://doi.org/10.1037/00223514.51.6.1173>

BARRENA-MARTÍNEZ， J.; LÓPEZ-FERNÁNDEZ， M.; MÁRQUEZ-MORENO, C.; ROMERO-FERNÁNDEZ, P. M. Corporate social responsibility in the process of attracting college graduates. Corporate Social Responsibility and Environmental Management, v. 22, n. 6, p. 408-423, 2015. 〈https://doi.org/10.1002/csr.1355>

BEATO, R. S.; SOUZA, M. T. S.; PARISOTTO, I. R. dos S. Rentabilidade dos índices de sustentabilidade empresarial em bolsas de valores: um estudo do ISE/Bovespa. RAI: Revista de Administração e Inovação, v. 6, n. 3, p. 108-127, 2009. 
BOAVENTURA, J. M. G.; SILVA, R. S. da; BANDEIRA DE MELLO, R. Corporate Financial Performance and Corporate Social Performance: methodological development and the theoretical contribution of empirical studies. Revista Contabilidade \& Finanças, v. 23, n. 60, p. 232-245, 2012. <http://dx. doi.org/10.1590/S1519-70772012000300008>

BRANCO, M. C.; RODRIGUES, L. L. Corporate social responsibility and resource-based perspectives. Journal of Business Ethics, v. 69, n. 2, p. 111-132, 2006. <https://doi.org/10.1007/s10551-006-9071-z>

BRITO, R. P.; BRITO, L. A. L. Vantagem competitiva, criação de valor e seus efeitos sobre o desempenho. Revista de Administração de Empresas, v. 52, n. 1, p. 70, 2012.

BRITO, R. P.; OLIVEIRA, L. B. A relação entre gestão de recursos humanos e desempenho organizacional. Brazilian Business Review, v. 13, n. 3, p. 94, 2016. <https://doi.org/10.15728/bbr.2016.13.3.5>

BRUNTON, M.; EWEJE, G.; TASKIN, N. Communicating corporate social responsibility to internal stakeholders: walking the walk or just talking the talk?. Business Strategy and the Environment, 2015. <https://doi.org/10.1002/bse.1889>

CARROLL, A. B. A commentary and an overview of key questions on corporate social performance measurement. Business \& Society, v. 39, n. 4, p. 466-478, 2000. <https://doi.org/10.1177/000765030003900406>

CARROLL, A. B. A three-dimensional conceptual model of corporate performance. Academy of Management Review, v. 4, n. 4, p. 497-505, 1979. <https://doi.org/10.5465/amr.1979.4498296>

CHAN, C.; CHOU, D.; LO, H. Do financial constraints matter when firms engage in CSR?. The North American Journal of Economics and Finance, v. 39, p. 241-259, 2017. <https://doi.org/10.1016/j.najef.2016.10.009>

CHENG, B.; IOANNOU, I.; SERAFEIM, G. Corporate social responsibility and access to finance. Strategic Management Journal, v. 35, n. 1, p. 1-23, 2014. <https://doi.org/10.1002/smj.2131>

COLLIER, J.; ESTEBAN, R. Corporate social responsibility and employee commitment. Business Ethics: A European Review, v. 16, n. 1, p. 19-33, 2007. <https://doi.org/10.1111/j.1467-8608.2006.00466.x>

CRISÓSTOMO, V. L.; FREIRE, F. S.; VASCONCELlOS, F. C. Corporate social responsibility, firm value and financial performance in Brazil. Social Responsibility Journal, v. 7, n. 2, p. 295-309, 2011. <https://doi.org/10.1108/17471111111141549>

DE BENEDiCTO, S. C.; STIEG, C. M.; LAMES, E. R.; SILVA FILHO, C. F. Responsabilidade social e estratégia em instituições financeiras públicas e privadas. Contextus - Revista Contemporânea de Economia e Gestão, v. 11, n. 1., 2013. <https://doi.org/10.19094/contextus. v11i1.32159> 
DIERICKX, I.; COOL, K. Asset stock accumulation and sustainability of competitive $\begin{array}{llllllll}\text { advantage. Management Science, } & \text { v. } 35, \quad \text { n. } 12, & \text { p. } & 1504-1511,\end{array}$ <https://doi.org/10.1287/mnsc.35.12.1504>

FREGUETE, L. M.; NOSSA, V.; FUNCHAL, B. Responsabilidade Social Corporativa e Desempenho Financeiro das Empresas Brasileiras na Crise de 2008. Revista de Administração Contemporânea, v. 19, n. 2, p. 232, 2015. <http://dx.doi.org/10.1590/19827849rac20151873>

FRÍAS-ACEITUNO, J. V.; RODRÍGUEZ-ARIZA， L.; GARCÍA-SÁNCHEZ， I. M. Is integrated reporting determined by a country's legal system? An exploratory study. Journal of Cleaner Production, v. 44, p. 45-55, 2013. <https://doi.org/10.1016/j.jclepro. 2012.12.006>

FRIEDMAN, M. The social responsibility of business is to increase its profits. The New York Times Magazine, 13 September. 1970.

GODFREY, P. C. The relationship between corporate philanthropy and shareholder wealth: A risk management perspective. Academy of Management Review, v. 30, n. 4, p. 777-798, 2005. <https://doi.org/10.5465/amr.2005.18378878>

GOLDNER NUNES, J.; TEIXEIRA, A. J. C.; NOSSA, V.; GALDI, F. C. Análise das variáveis que influenciam a adesão das empresas ao índice BM\&F Bovespa de sustentabilidade empresarial. Revista Base (Administração e Contabilidade) da Unisinos, v. 7, n. 4, 2010. <http://doi.org/10.4013/base.2010.74.06>

GRECCO, M. C. P.; GERON, C. M. S; GRECCO, G. B. Corporate social responsibility and its relation with performance and earnings management. Contabilidade Vista \& Revista, v. 28, n. 1, p. 25-44, 2017.

GRIFFIN, J. J. Corporate social performance: Research directions for the 21 st century. $\begin{array}{llllllll}\text { Business \& Society, } & \text { v. } 39, \quad \text { n. } & 4, & \text { p. } & 479-491, & \end{array}$ <https://doi.org/10.1177/000765030003900407>

GRIFFIN, J. J.; MAHON, J. F. The corporate social performance and corporate financial performance debate: Twenty-five years of incomparable research. Business \& Society, v. 36, n. 1, p. 5-31, 1997. <https://doi.org/10.1177/000765039703600102>

GVCes. Índice de Sustentabilidade Empresarial. Centro de Estudos em Sustentabilidade da EAESP. Disponível em: <http://www.isebvmf.com.br>. Acesso em 13/06/2014.

HAIR, J. F.; BLACK, W. C.; BABIN, B. J.; ANDERSON, Rolph E.; TATHAM, Ronald L. Análise multivariada de dados. Bookman Editora, 2009.

HART, S. L.; DOWELL, G. Invited editorial: A natural-resource-based view of the firm fifteen years after. Journal of Management, v. 37, n. 5, p. 1464-1479, 2011. <https://doi.org/10.1177/0149206310390219>

HILLMAN, A. J.; KEIM, G. D. Shareholder value, stakeholder management, and social issues: what's the bottom line?. Strategic Management Journal, p. 125-139, 2001. <https://doi.org/10.1002/1097-0266(200101)22:2<125::AID-SMJ150>3.0.CO;2-H> 
HUSELID, M. A. The impact of human resource management practices on turnover, productivity, and corporate financial performance. Academy of Management Journal, v. 38, n. 3, p. 635-672, 1995. <https://doi.org/10.5465/256741>

HUSTED, B. W.; ALLEN, D. B.; KOCK, N. Value creation through social strategy. Business \& Society, v. 54, n. 2, p. 147-186, 2015. <https://doi.org/10.1177/0007650312439187>

JOIA, L. A.. Measuring intangible corporate assets: linking business strategy with intellectual capital. Journal of Intellectual Capital, v. 1, n. 1, p. 68-84, 2000. <https://doi.org/10.1108/14691930010371636>

KARNANI, A. "Doing well by doing good": The grand illusion. California Management Review, v. 53, n. 2, p. 69-86, 2011.

KAYO, E. K. Organizational Growth, Firm Size and Economic Value. Revista de Administração Contemporânea, v. 19, n. $1, \quad$ p. $142-148,2015$. <http://dx.doi.org/10.1590/1982-7849rac20158121>

KAYO, E. K.; KIMURA, H.; MARTIN, D. M. L.; NAKAMURA; W. T. Ativos intangíveis, ciclo de vida e criação de valor. Revista de Administração Contemporânea, v. 10, n. 3, p. 73-90, 2006. <http://doi.org/10.1590/S1415-65552006000300005>

LAZONICK, W.; O'SULLIVAN, M. Maximizing shareholder value: a new ideology for corporate governance. Economy and Society, v. 29, n. 1, p. 13-35, 2000. <https://doi.org/10.1080/030851400360541>

LUO, X.; WANG, H.; RAITHEL, S.; ZHENG, Q. Corporate social performance, analyst stock recommendations, and firm future returns. Strategic Management Journal, v. 36, n. 1, p. 123136, 2015. <https://doi.org/10.1002/smj.2219>

MACHADO, M. R.; MACHADO, M. A. V.; CORRAR, L. J. Desempenho do índice de sustentabilidade empresarial (ISE) da Bolsa de Valores de São Paulo. Revista Universo Contábil, v. 5, n. 2, p. 24-38, 2009. <https://doi.org/10.4270/ruc.2009211>

MADORRAN, C.; GARCIA, T. Corporate social responsibility and financial performance: the Spanish case. Revista de Administração de Empresas, v. 56, n. 1, p. 20-28, 2016. <http://dx.doi.org/10.1590/S0034-759020160103>

MAHON, J.; WARTICK, S. L. Corporate social performance profiling: using multiple stakeholder perceptions to assess a corporate reputation. Journal of Public Affairs, v. 12, n. 1, p. 12-28, 2012. 〈https://doi.org/10.1002/pa.433>

MARGOLIS, J. D.; WALSH, J. P. Misery loves companies: Rethinking social initiatives by business. Administrative Science Quarterly, v. 48, n. 2, p. 268-305, 2003. <https://doi.org/10.2307/3556659>

MARTINUZZI, A.; KRUMAY, B. The good, the bad, and the successful-how corporate social responsibility leads to competitive advantage and organizational transformation. Journal of 
$\begin{array}{llllllll}\text { Change Management, } & \text { v. } 13, \quad \text { n. } & 4, & \text { p. } & 424-443,\end{array}$ <https://doi.org/10.1080/14697017.2013.851953>

MAZZIONI, S.; DIEL, F. J.; DIEL, E. H.; KRUGER, S. D.; KLANN, R. C. Análise dos Indicadores de valor adicionado das empresas participantes do Índice de Sustentabilidade Empresarial (ISE) e das demais empresas listadas na BM\&FBovespa. Contextus - Revista Contemporânea de Economia e Gestão, v.11, n. 2, p. 159-180, 2013. <https://doi.org/10.19094/contextus. v11i2.527>

MCWILlIAMS, A.; SIEGEL, D. S. Corporate Social Responsibility and Financial Performance: Correlation or Misspecification? Strategic Management Journal, v. 21, n. 5, p. 603-609, 2000. <https://doi.org/10.1002/(SICI)1097-0266(200005)21:5<603::AID$\mathrm{SMJ} 101>3.0 . \mathrm{CO} ; 2-3>$

MCWILLIAMS, A.; SIEGEL, D. S. Creating and capturing value: Strategic corporate social responsibility, resource-based theory, and sustainable competitive advantage. Journal of Management, v. 37, n. 5, p. 1480-1495, 2011. <https://doi.org/10.1177/0149206310385696>

MELO, D. N. B.; ROMERO, C. B. A.; REINADO, H. O. A.; ABREU, C. B. Sustentabilidadeuma investigação da atitude e do comportamento de estudantes de administração. Contextus Revista Contemporânea de Economia e Gestão, v. 16, n. 1, p. 34-61, 2018. $<$ https://doi.org/10.19094/contextus.v0i0.33311>

MELO, E. C. de; ALMEIDA, F. M. de; SANTANA, G. A. da S. Índice de sustentabilidade empresarial (ISE) e desempenho financeiro das empresas do setor de papel e celulose. Revista Contabilidade e Controladoria, v. 4, n. 3, 2012. <http://dx.doi.org/10.5380/rcc.v4i3.30188>

NELLING, E.; WEBB, E. Corporate social responsibility and financial performance: the "virtuous circle" revisited. Review of Quantitative Finance and Accounting, v. 32, n. 2, p. 197-209, 2009. 〈https://doi.org/10.1007/s11156-008-0090-y>

ONKILA, T. Pride or embarrassment? Employees' emotions and corporate social responsibility. Corporate Social Responsibility and Environmental Management, v. 22, n. 4, p. 222-236, 2015. <https://doi.org/10.1002/csr. 1340>

ORELLANO, V. I. F.; QUIOTA, S. Análise do Retorno dos Investimentos Socioambientais das Empresas Brasileiras. Revista de Administração de Empresas, v. 51, n. 5, p. 471, 2011.

ORLITZKY, M.; SCHMIDT, F. L.; RYNES, S. L. Corporate social and financial performance: A meta-analysis. Organization Studies, v. 24, n. 3, p. 403-441, 2003. <https://doi.org/10.1177/0170840603024003910>

ORSATO, R. J.; CAMPOS, J. G. F. de; BARAKAT, S. R.; NICOLLETTI, M.; MONZONI, M. Why join a carbon club? A study of the banks participating in the Brazilian "Business for Climate Platform". Journal of Cleaner Production, v. 96, p. 387-396, 2015. <https://doi.org/10.1016/j.jclepro.2014.01.007>

RAITHEL, S.; SCHWAIGER, M. The effects of corporate reputation perceptions of the general public on shareholder value. Strategic Management Journal, v. 36, n. 6, p. 945-956, 2015. <https://doi.org/10.1002/smj.2248> 
RAMSEY, J. B. Tests for specification errors in classical linear least-squares regression analysis. Journal of the Royal Statistical Society: Series B (Methodological), v. 31, n. 2, p. 350-371, 1969. <https://doi.org/10.1111/j.2517-6161.1969.tb00796.x>

RIBEIRO, H. C. M. Teoria dos stakeholders: um estudo bibliométrico de sua produção acadêmica divulgada nos periódicos nacionais de 1999 a 2013. Contextus - Revista Contemporânea de Economia e Gestão, v. 14, n. 1, p. 163-192, 2016. <https://doi.org/10.19094/contextus.v14i1.810>

ROBERTS, P. W.; DOWLING, G. R. Corporate reputation and sustained superior financial performance. Strategic Management Journal, v. 23, n. 12, p. 1077-1093, 2002. <https://doi.org/10.1002/smj.274>

RUF, B. M.; MURALIDHAR, K.; BROWN, R. M.; JANNEY, J. J.; PAUL, K. An empirical investigation of the relationship between change in corporate social performance and financial performance: A stakeholder theory perspective. Journal of Business Ethics, v. 32, n. 2, p. 143 156, 2001. <https://doi.org/10.1023/A:1010786912118>

RUSSO, M. V.; FOUTS, P. A. A resource-based perspective on corporate environmental performance and profitability. Academy of Management Journal, v. 40, n. 3, p. 534-559, 1997. <https://doi.org/10.5465/257052>

SOUZA CUNHA, F. A. F.; SAMANEZ, C. P. Performance analysis of sustainable investments in the Brazilian stock market: a study about the corporate sustainability index (ISE). Journal of Business Ethics, v. 117, n. 1, p. 19-36, 2013. <https://doi.org/10.1007/s10551-012-1484-2>

SURROCA, J.; TRIBÓ, J. A.; WADDOCK, S. Corporate responsibility and financial performance: The role of intangible resources. Strategic Management Journal, v. 31, n. 5, p. 463-490, 2010. <https://doi.org/10.1002/smj.820>

TEIXEIRA, E. A.; NOSSA, V.; FUNCHAL, B. O índice de sustentabilidade empresarial (ISE) e os impactos no endividamento e na percepção de risco. Revista Contabilidade \& Finanças, v. 22, n. 55, p. 29-44, 2011. <https://doi.org/10.1590/S1519-70772011000100003>

TURBAN, D. B.; GREENING, Daniel W. Corporate social performance and organizational attractiveness to prospective employees. Academy of Management Journal, v. 40, n. 3, p. 658-672, 1997. <https://doi.org/10.5465/257057>

TURRA, S.; VERGINI, D. P.; JACOMOSSI, F. A.; HEIN, N. Efeitos do Capital Intelectual sobre o desempenho financeiro em empresas brasileiras e chilenas. Contextus - Revista Contemporânea de Economia e Gestão, v. 13, n. 2, p. 82-104, 2015. <https://doi.org/10.19094/contextus.v13i2.637>

ULLMANN, A. A. Data in search of a theory: A critical examination of the relationships among social performance, social disclosure, and economic performance of US firms. Academy of $\begin{array}{lllllll}\text { Management Review, } & \text { v. } 10, \quad \text { n. } 3, \quad \text { p. } & \text { 540-557, } & 1985 .\end{array}$ <https://doi.org/10.5465/amr.1985.4278989> 
VIEIRA, K. M.; KREUTZ, R. R. Endividado ou poupador: Impacto das variáveis sociodemográficas e do gerenciamento financeiro. Revista Pretexto, v. 19, n. 3, p. 81-98, 2018. <http://dx.doi.org/10.21714/pretexto.v19i3.5374>

WADDOCK, S. Parallel universes: Companies, academics, and the progress of corporate citizenship. Business and Society Review, v. 109, n. 1, p. 5-42, 2004. <https://doi.org/10.1111/j.0045-3609.2004.00002.x>

WADDOCK, S. A.; GRAVES, Samuel B. The corporate social performance-financial performance link. Strategic Management Journal, v. 18, n. 4, p. 303-319, 1997. <https://doi.org/10.1002/(SICI)1097-0266(199704)18:4<303::AID-SMJ869>3.0.CO;2-G>

WERNERFELT, B. A resource-based view of the firm. Strategic Management Journal, v. 5, n. 2, p. 171-180, 1984. <https://doi.org/10.1002/smj.4250050207> 